\title{
DOSAGE DU SODIUM, DU POTASSIUM, DU CALCIUM ET DU MAGNÉSIUM PAR SPECTROPHOTOMÉTRIE DE FLAMME DANS LES ALIMENTS, LE LAIT ET LES EXCRETA
}

\author{
L. GUEGUEN et P. ROMBAUTS \\ avec la collaboration technique de Ginette Lepine et Maryvonne Le GorF \\ Service de Biochimie et de Nutrition, \\ Centre national de Recherches zootechniques, Jouy-en-Josas.
}

\section{SOMMAIRE}

Le photomètre à flamme "Eppendorf "offre des possibilités nouvelles pour les dosages des métaux alcalins et alcalino-terreux. Il permet de doser dans de très bonnes conditions le sodium, le potassium et le calcium. Le dosage du magnésium, beaucoup plus délicat, est cependant possible grâce à l'emploi d'un photomultiplicateur d'électrons très sensible et d'une flamme d'hydrogène, dont l'émission propre est faible aux basses longueurs d'onde.

Bien que particulièrement sélectif, ce photomètre ne permet pas d'éliminer toutes les interférences, et l'influence du potassium en particulier est très marquée dans tous les dosages. De même, le sodium excite considérablement l'émission du magnésium. En revanche, l'action du calcium dans les dosages du potassium et du sodium est nulle, et l'influence du phosphore dans le dosage du calcium est négligeable.

Dans cette étude, les conditions optima à réunir pour l'analyse des fourrages, du lait, des fécès et de l'urine ont été définies, en ayant précisé les principales causes d'erreur rencontrées et justifié la validité des résultats obtenus.

\section{INTRODUCTION}

Ia spectrophotométrie de flamme offre, par rapport aux méthodes chimiques courantes, des possibilités intéressantes pour l'étude de la composition minérale de produits très variés. Elle permet en particulier de doser le sodium, le potassium, le calcium et le magnésium, avec une bonne précision, à des concentrations très faibles ; par sa rapidité, elle se prête parfaitement aux dosages en série.

Cependant l'application de la spectrophotométrie de flamme pose souvent des problèmes complexes et il importe de s'attacher en premier lieu au contrôle de la validité des résultats obtenus. Malgré la bonne sélectivité des appareils utilisés, il existe toujours des interférences dues à d'autres éléments présents dans la solution. Le sens et la grandeur de ces interférences varient bien entendu suivant la composition du produit à analyser, mais également suivant l'appareil utilisé. C'est pourquoi il nous 
a paru nécessaire de faire une mise au point particulière au photomètre «Eppendorf »* que nous utilisons, et pour les principaux produits qui nous intéressent (aliments, lait, fécès, urine). Nous avons étudié systématiquement les interférences dues aux principaux éléments présents dans ces produits et, les connaissant, nous avons défini pour chaque dosage les conditions optima permettant de les éliminer.

\section{I. - CaRActÉRistigues du PHOTOMÈTre A FLAMME « EPPENDORF ».}

- Le photomètre à flamme « Eppendorf » permet d'utiliser plusieurs carburants (propane, acétylène, hydrogène) et comporte une admission éventuelle d'oxygène. Il est donc possible d'obtenir une flamme chaude permettant l'excitation du calcium et du magnésium. Les pressions des différents gaz peuvent être réglées avec une bonne précision et la flamme obtenuze est très stable. Le tableau I résume les principales caractéristiques des flammes utilisées.

\section{TABLEAU I}

Caractíristiques des flammes.

\begin{tabular}{|c|c|c|c|}
\hline $\mathrm{Gaz}$ & $\begin{array}{l}\text { Températures } \\
\text { de flamme en }{ }^{\circ} \mathrm{C}\end{array}$ & $\begin{array}{l}\text { Pressions } \\
\text { du carburant } \\
\text { en } m m \text { d'eau }\end{array}$ & $\begin{array}{l}\text { Débits } \\
\text { du carburant } \\
\text { en litres/mn }\end{array}$ \\
\hline $\begin{array}{l}\text { Propane-air ...... } \\
\text { Acétylène-air ..... } \\
\text { Acétylène-air-oxy - } \\
\text { gène ........... } \\
\text { Hỹdrogène-air-oxy- } \\
\text { gène.......... }\end{array}$ & $\begin{array}{l}1925 \\
2400 \\
3100 \\
2600\end{array}$ & $\begin{array}{l}160 \\
500 \\
500 \\
800\end{array}$ & $\begin{array}{l}0,5 \\
1\end{array}$ \\
\hline
\end{tabular}

- L'utilisation de l'hydrogène permet d'améliorer la sensibilité du dosage de $\mathrm{Mg}$, dont l'émission est mesurée à une courte longueur d'onde. Fin effet les autres gaz (propane et acétylène) présentent dans cette zone du spectre des bandes d'émission dues au carbone, tandis que l'émission propre de l'hydrogène y est beaucoup plus faible.

- Un dispositif de chambre d'atomisation très bien conçu assure une pulvérisation très fine du liquide, et le débit est particulièrement faible et régulier.

- L'utilisation d'un système de filtres interférentiels, à bande passante très étroite, permet une bonne sélectivité.

- Il existe trois échelles de sensibilité et, d'autre part, 1'appareil est muni d'un photomultiplicateur d'électrons à 9 étages. Ceci permet une grande possibilité dans le choix des concentrations à utiliser pour les dosages.

L'avantage de cet appareil est de permettre les dosages de Ca et de Mg qui, en général, en raison des nombreuses interférences et du manque de sensibilité, ne peuvent être effectués à l'aide des autres appareils. En ce qui concerne $\mathrm{Na}$ et $\mathrm{K}$, un photomètre à flamme de modèle plus simple (Electrosynthèse) nous a đonné des résultats très comparables à ceux obtenus à l'aide de l'appareil étudié.

* Fabricants : Netheler et Hinz, Hambourg

Annales de Biologie animale. I-I $96 \mathrm{r}$ 


\section{II. - ÉTUDE DES INTERFÉRENCES}

L'étude de toutes les interférences susceptibles de se produire dans l'analyse par photométrie de flamme offre un champ de recherche inépuisable. Aussi, avons nous limité nos investigations aux interférences susceptibles de perturber les dosages dans les milieux qui intéressent le nutritionniste.

\section{A - Influence du milieu organique}

Les substances organiques nuisent à la précision et à la fidélité du dosage en modifiant les propriétés physiques de la solution, en particulier sa viscosité, sa densité et sa tension superficielle. Elles contribuent donc à réduire la quantité de liquide atteignant la flamme sous forme d'aérosol, en diminuant la quantité de liquide consommée et en abaissant son taux d'atomisation. De plus, les modifications des propriétés physiques du liquide influent sur les dimensions des gouttes et, en conséquence, sur les conditions d'excitation des éléments.

La matière organique présente (liquide biologique non minéralisé) ou ajoutée (acide trichloracétique utilisé pour la déprotéinisation du lait) nuit ainsi à la fidélité de la méthode (en provoquant une pulvérisation et une émission irrégulières) et à son exactitude, lorsque les solutions étalons utilisées ne possèdent pas les mêmes propriétés physiques.

Ce problème est très important puisque sa résolution peut permettre de savoir si, dans le cas de liquides biologiques (lait, urine...) il est nécessaire d'effectuer une minéralisation préalable.

Cependant, afin d'éviter l'élimination des substances tensio-actives, il est possible d'effectuer les dosages en diluant suffisamment les solutions ou en ajoutant des substances qui réduisent la tension superficielle (par exemple l'alcool). Dans le cas de l'urine par exemple, en diluant de 50 à 200 fois, il est possible de doser $\mathrm{Na}$ et $\mathrm{K}$ dans de bonnes conditions. En revanche, pour le lait, de fortes dilutions ne permettent pas d'obtenit une consommation et une pulvérisation normales du liquide; il est au moins nécessaire d'effectuer alors une défécation préalable. Ainsi, après défécation à l'aide de 1'acide trichloracétique par exemple, on obtient des résulats convenables pour $\mathrm{Na}, \mathrm{K}, \mathrm{Ca}$. Cependant, afin d'obtenir le maximum de fidélité et d'exactitude, en particulier pour le dosage de $\mathrm{Mg}$, il est nécessaire de minéraliser.

\section{$\mathrm{B}$ - Influence des anions}

Les anions, présents dans le produit ou introduits pendant la préparation de la solution sous forme d'acides libres, agissent sur l'émission de l'élément à doser en réduisant 1'ionisation des cations dans la flamme. Selon BakER et Johnson (I954), l'action de l'ion $\mathrm{H}^{+}$est négligeable par rapport à celle des anions et, de toute façon, l'acidité du milieu ne serait gênante que pour un $\mathrm{pH}$ inférieur à 2 , où il se produirait une légère diminution de 1'émission des alcalins.

Nous avons particulièrement étudié l'action des anions apportés par les acides utilisés dans la préparation des solutions, ou demeurant dans le résidu de minéralisation par voie humide.

L'acide nitrique et l'acide chlorhydrique sont souvent utilisés pour redissoudre 
le résidu d'incinération, et leur action, résumée par les graphiques suivants, se caractérise par une faible inhibition dans tous les dosages (figure I).
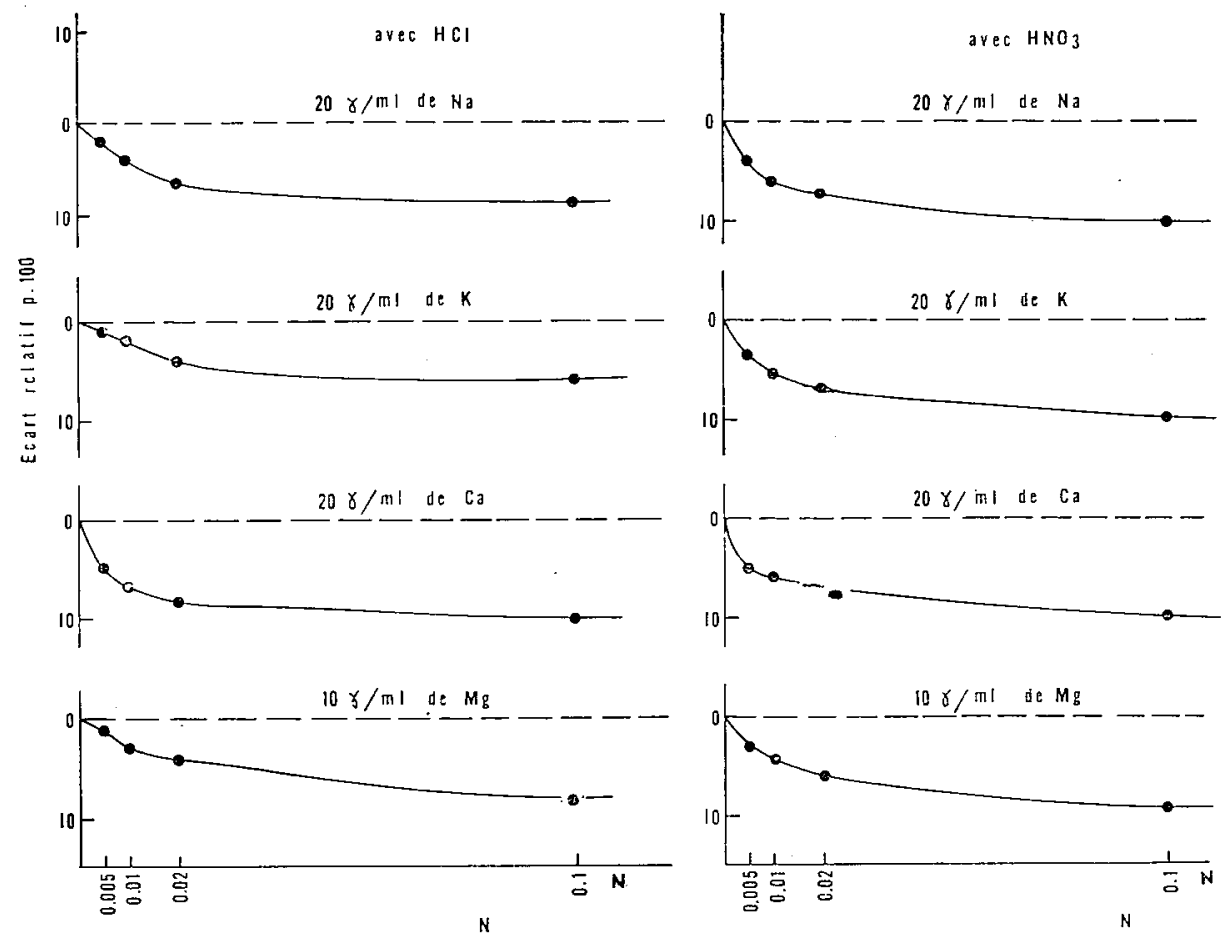

FIG. x. - Interférences dues aux acides $\mathrm{HCl}_{\text {el }} \mathrm{HNO}_{3}$ dans les dosages de $\mathrm{K}, \mathrm{Na}, \mathrm{Ca}, \mathrm{Mg}$.

L'acide perchlorique demeure dans le résidu de minéralisation nitro-perchlorique; il exerce une action inhibitrice très élevée, résumée dans le tableaı 2.

\section{TABLEAU 2}

Interférences dues à l'acide perchlorique.

\begin{tabular}{|c|c|c|c|}
\hline Élément dosé & $\begin{array}{c}\gamma / \mathrm{ml} \text { de l'élément } \\
\text { à doser }\end{array}$ & $\begin{array}{c}\text { Concentration } \\
\text { du milieu en } \mathrm{HClO}_{4}\end{array}$ & $\begin{array}{c}\text { Lectures } \\
\text { (en divisions) }\end{array}$ \\
\hline $\mathrm{K}$ & $\begin{array}{l}30 \\
30\end{array}$ & $\begin{array}{l}\text { Néant } \\
0,6 \mathrm{~N}\end{array}$ & $\begin{array}{l}280 \\
198\end{array}$ \\
\hline $\mathrm{Na}$ & $\begin{array}{l}20 \\
20\end{array}$ & $\begin{array}{l}\text { Néant } \\
0,6 \mathrm{~N}\end{array}$ & $\begin{array}{l}90 \\
70\end{array}$ \\
\hline $\mathrm{Ca}$ & $\begin{array}{l}40 \\
40\end{array}$ & $\begin{array}{l}\text { Néant } \\
0,6 \mathrm{~N}\end{array}$ & $\begin{array}{l}280 \\
220\end{array}$ \\
\hline
\end{tabular}

En conséquence, il est indispensable de connaître avec précision les quantités d'acide perchlorique utilisées, et d'ajouter des quantités équivalentes dans les solutions étalons. 
D'autre part, dans le cas particulier du potassium, la solubilité de $\mathrm{KClO}_{4}$ dans un milieu contenant $\mathrm{HClO}_{4}$ est différente de sa solubilité dans 1'eau ; le tableau 3 indique la limite de solubilité.

\section{TABLEAU 3}

Mesure de la solubilité du perchlorale de $K$.

\begin{tabular}{c|c|c}
\hline \hline & K ajouté & K apparent mesuré \\
\cline { 2 - 2 } & $500 \gamma / \mathrm{ml}$ de $\mathrm{K}$ & 500 \\
" & $1,4 \mathrm{~N}$ & 1000 \\
" & $2000 \mathrm{\gamma} / \mathrm{ml}$ de $\mathrm{K}$ & 1025 \\
$3000 \mathrm{ml}$ de $\mathrm{K}$ & 1025 \\
\hline
\end{tabular}

Ainsi la solubilité de $\mathrm{KClO}_{4}$ est de I g par litre dans $\mathrm{HClO}_{4}$, tandis qu'elle est de $2,5 \mathrm{~g} /$ litre dans l'eau. Ceci peut constituer une cause d'erreur pour de fortes concentrations en $\mathrm{K}$.

La difficulté résultant de ces interférences est facile à surmonter, puisque 1'on connait avec une précision suffisante les quantités d'acides se trouvant dans la solution (dans le cas de la minéralisation nitro-perchlorique à $220-250^{\circ} \mathrm{C}, 1^{\prime}$ acide nitrique est chassé, tandis que la totalité de l'acide perchlorique demeure dans la solution). Il suffit donc de compenser cette action en ajoutant les mêmes quantités dans les solutions étalons.

L'action dépressive de l'ion $\mathrm{PO}_{4}^{----}$dans les dosages du calcium et des alcalins a été souvent étudiée, en raison de la présence constante de $\mathrm{P}$ dans les milieux biologiques. Cependant, en utilisant la flamme d'acétylène (avec ou sans oxygène) nous avons constaté une action pratiquement nulle du phosphore aux concentrations habituelles (voir fig. 3). Cette influence négligeable du phosphore sur l'émission du calcium serait une caractéristique de l'appareil et proviendrait surtout de l'emploi de la chambre de pulvérisation : en effet, la suppression de cette chambre entraîne une action dépressive très marquée du phosphore sur l'émission de Ca.

L'acide trichloracétique, contrairement aux autres acides, augmente l'émission de tous les éléments, ce qui peut s'expliquer par l'action combinée de l'anion luimême et de la molécule organique qui modifie la tension superficielle. Il est facile d'utiliser pour l'analyse du lait déprotéinisé des solutions étalons contenant de l'acide trichloracétique.

\section{$\mathrm{C}$ - Influence des cations}

Les cations présents dans la solution infuent sur le dosage de l'élément à mesurer par leur émission propre, due au fait que plusieurs cations sont plus ou moins fortement excités dans la flamme simultanément et sous l'influence des mêmes conditions. Ceci constitue les interférences vraies, principales causes d'erreur en photométrie de flamme.

Nous distinguerons, comme le font BuRRIEL-MARTI et RAMIREZ-MunOz (I957), les interférences dues à des radiations de même longueur d'onde que celle de l'élément à doser (interférences directes) et celles dues à la présence d'éléments émettant des radiations de longueur d'onde très différente. 
Parmi les interférences directes il faut citer l'influence du fond de flamme et des spectres continus. Certaines interférences directes sont considérablement réduites par l'emploi de filtres à bande passante très étroite et, grâce aut photomulitiplicateur d'électrons utilisé au lieu des cellules courantes, la sensibilité des dosages n'est pas réduite. Notons également que la présence de substances organiques dans la solution accentue l'interférence due au fond de flamme.

Nous considérerons successivement dans chacun des dosages ( $\mathrm{Na}, \mathrm{K}, \mathrm{Ca}, \mathrm{Mg}$ ) les interférences dues aux principaux éléments rencontrés dans les produits étudiés.

I Dosage du potassium : le potassium est en général l'élément le plus abondant dans les produits végétaux, le lait et l'urine des ruminants, et peu de problèmes d'interférences se posent pour son dosage.

On utilise la raie caractéristique du potassium à $768 \mathrm{~m} \mu$, et aucune interférence directe ne se produit. Comme l'indique le tableau 4 , même à des concentrations doubles, le calcium n'a pratiquement aucune influence sur le dosage du potassium.

TABLEAU 4

Interférences due à Ca dans le dosage de $K$.

\begin{tabular}{c|c|c}
\hline \hline K en $\gamma / \mathrm{ml}$ & Ca ajouté en $\gamma / \mathrm{ml}$ & Lectures \\
\hline 10 & 0 \\
0 & 49. \\
0 & 106 \\
20 & $\mathbf{2 8 0}$ \\
\hline 50 & 10 \\
& 10 \\
10 & 50 \\
20 & 10 & 205 \\
50 & 10 & 288 \\
\hline 10 & 15 & 50,0 \\
10 & 20 & $\mathbf{5 0 , 5}$ \\
10 & 50,0 \\
\hline \hline
\end{tabular}

$2^{\circ}$ Dosage du sodium: le filtre utilisé a son maximum de transmission à $589 \mathrm{~m} \mu$. I'ion le plus gênant, aux concentrations courantes, est le potassium, provoquant

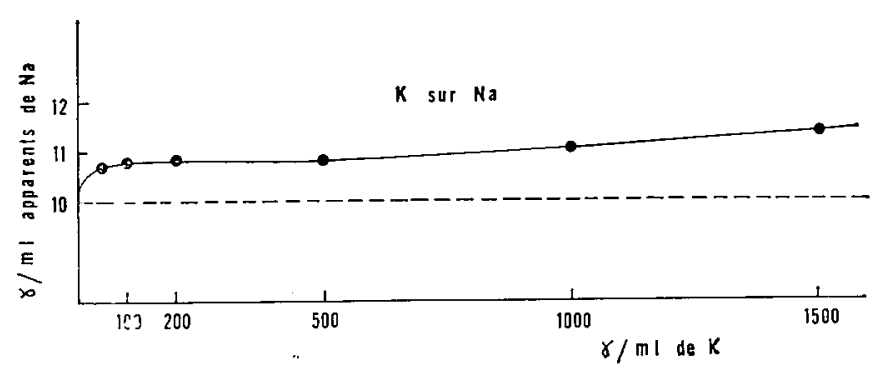

FIG. 2. - Interférence due à $K$ dans le dosage de $N a$.

ici une interférence indirecte, sans doute par une interaction ayant lieu dans la flamme et troublant les conditions d'excitation du sodium. Comme le montre la figure 2 , nous obtenons une augmentation brutale de l'émission de $\mathrm{Na}$, suivie d'un palier pour 
les concentrations moyennes en $\mathrm{K}$. Il suffit donc, si la composition du produit est mal connue, d'obtenir une interférence maximum en ajoutant dans la solution et dans les témoins une forte quantité de $\mathrm{K}$. Plus simplement, il suffit de doser $\mathrm{K}$ avant Na.

Le calcium à de fortes concentrations peut inhiber sensiblement l'émission du sodium, comme le montre le tableau 5 .

TABLEAU 5

Interférence due à $\mathrm{Ca}$ dans le dosage de $\mathrm{Na}$.

\begin{tabular}{c|c|c}
\hline \hline Na en $\gamma / \mathrm{ml}$ & Ca ajouté en $\gamma / \mathrm{ml}$ & Lectures \\
\cline { 1 - 2 } 2,5 & 0 & 26 \\
5 & 0 & 45 \\
10 & 0 & 90 \\
\hline 2,5 & 5 & 26 \\
5 & 5 & 47 \\
10 & 5 & 90 \\
\hline 2,5 & 10 & 24,0 \\
2,5 & 15 & 23,5 \\
2,5 & 20 & 23,0 \\
\hline \hline
\end{tabular}

Notons également que 1'ion $\mathrm{NH}_{4}{ }^{+}$excite considérablement l'émission de $\mathrm{Na}$, puisque dans un milieu $\mathrm{NH}_{4} \mathrm{OH} N$ nous trouvons pour le dosage de $\mathrm{Na}$ une erreur par excès atteignant $35 \mathrm{p}$. roo. Cependant ceci ne peut constituer une cause importante d'erreur, car, même dans le cas d'une minéralisation nitro-perchlorique, la solution à analyser ne contient plus que des traces d'azote.

\section{TABLEAU 6}

Interférences dues à $\mathrm{K}$ et $\mathrm{Na}$ dans le dosage de $\mathrm{Ca}$.

\begin{tabular}{c|cc}
\hline $\begin{array}{c}\text { Quantité de Ca } \\
\text { en } \gamma / \mathrm{ml}\end{array}$ & $\begin{array}{c}\text { Quantités de K ou Na } \\
\text { ajoutées, en } \gamma / \mathrm{ml}\end{array}$ & $\begin{array}{c}\text { Ca apparent mesuré } \\
\text { en } \gamma / \mathrm{ml}\end{array}$ \\
\hline 50 & $0 \mathrm{~K}$ & 50,0 \\
50 & 200 & 48,3 \\
50 & 500 & 47,9 \\
50 & 1000 & 47,8 \\
50 & 3000 & 47,9 \\
50 & 4000 & 47,9 \\
50 & 5000 & 47,9 \\
\hline 50 & $0 \mathrm{Na}$ & 50,0 \\
50 & 200 & 49,8 \\
50 & 400 & 50,0 \\
50 & 800 & 50,0 \\
50 & 1200 & 50,0 \\
50 & 1600 & 50,0 \\
\hline \hline
\end{tabular}


$3^{\circ}$ Dosage du calcium : nous utilisons le filtre correspondant à la bande présentant un maximum à $623 \mathrm{~m} \mu$. Dans cette zone, 1'émission de Ca pourrait être accrue par une interférence due à un fond continu de $\mathrm{Na}$ et $\mathrm{K}$. En fait, comme le montre le tableau 6 , seul $\mathrm{K}$ peut avoir une action non négligeable, dans le sens d'une diminution de l'émission, due ainsi à une interférence indirecte.

Ainsi, même à des concentrations très élevées (rencontrées en particulier dans l'urine), l'interférence due à $\mathrm{Na}$ est nulle.

Rappelons que l'action du phosphore est également très faible, comme le montre la figure 3, qui résume d'autre part nos résultats concernant les interférences dues à $\mathrm{Al}, \mathrm{Fe}, \mathrm{Mg}$.
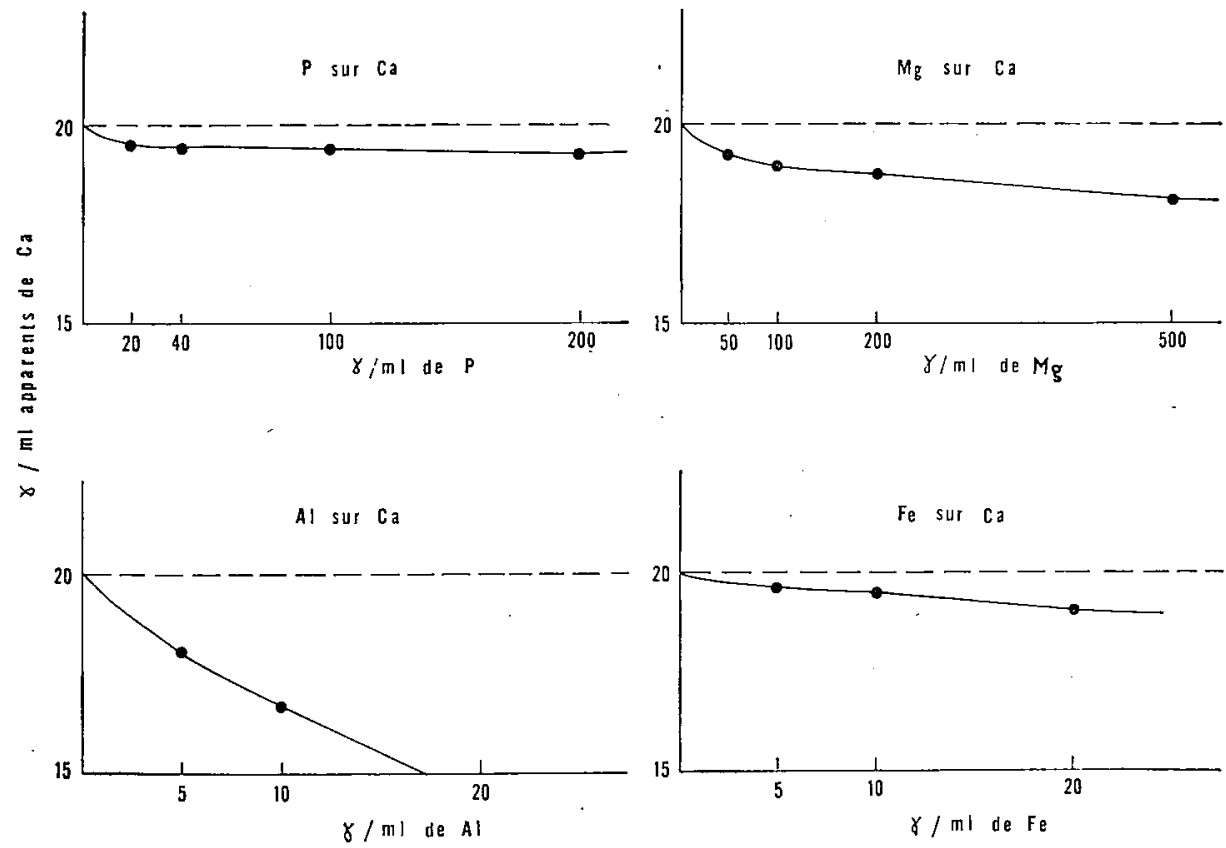

FIG. 3. - Principales interférences dans le dosage du calcium.

Selon Mitchel, et RobERTSON (I936) l'action de 1'aluminium, particulièrement importante, pourrait être éliminée en ajoutant dans les solutions à analyser et dans les témoins une certaine quantité de strontium. Cependant, à la longureur d'onđe du filtre que nous utilisons (maximum de $623 \mathrm{~m} \mu$ ) l'interférence propre due au strontium est importante et cette méthode ne peut être appliquée sans diminuer la précision des mesures. C'est pourquoi nous avons utilisé pour ces essais un filtre interférentiel à $554 \mathrm{~m} \mu$, longueur d'onde où l'émission propre du strontium est faible.

Comme le montre la figure 3 bis, il est possible, en ajoutant dans toutes les solutions un grand excès de $\mathrm{Sr}$, de réduire considérablement 1'inhibition due à $\mathrm{Al}$ et de mesurer la teneur en $\mathrm{Ca}$ avec une précision satisfaisante quand le rapport $\frac{\mathrm{A} 1}{\mathrm{Ca}}$ atteint des valeurs élevées.

$4^{0}$ Dosage du magnésium : la maximum de transmission du filtre utilisé est à $380 \mathrm{~m} \mu$ (sommet d'une bande), et dans la mesure de l'émission du magnésium on 
devrait se heurter à plusieurs interférences directes, en particulier dues au fer, au manganèse et peut-être au potassium.

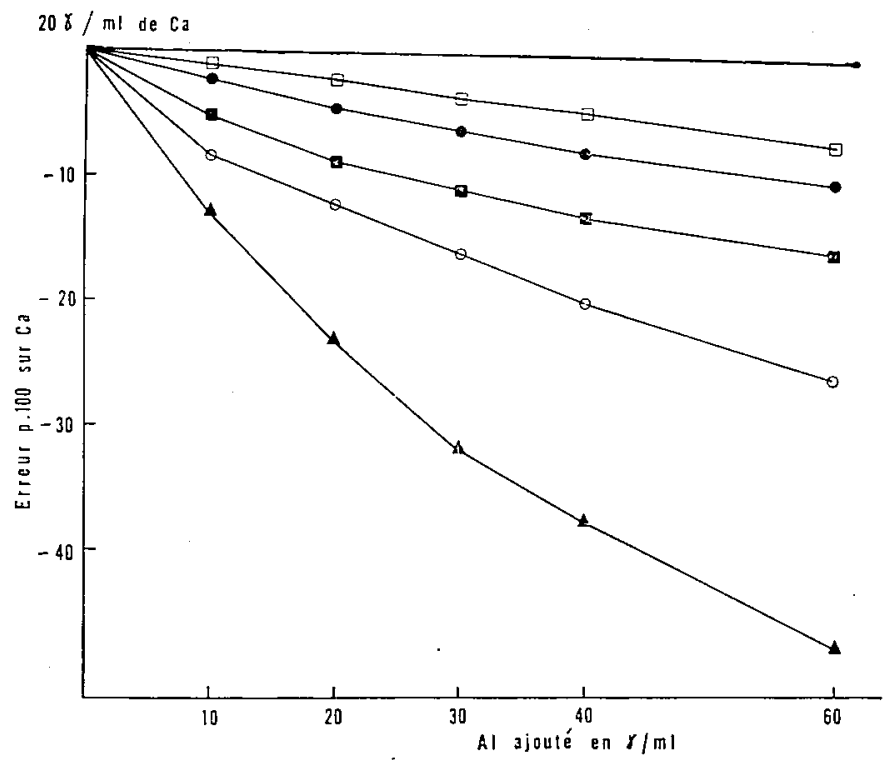

FIG. 3 bis. - Diminution, par addition de strontiun, de l'inhibition due à laluminium dans le dosage du calcium.

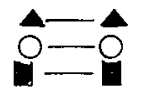

\section{sans $\mathrm{Sr}$}

avec $200 \gamma / \mathrm{ml}$ de $\mathrm{Sr}$ avec $500 \mathrm{\gamma} / \mathrm{ml}$ de $\mathrm{Sr}$.

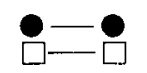

avec rooo $\mathrm{\gamma} / \mathrm{ml}$ de $\mathrm{Sr}$. avec $2000 \gamma / \mathrm{ml}$ de $\mathrm{Sr}$.

Le tableau 7 montre que Fe et $M n$ n'augmentent l'émission de $M g$ qu'à partir de concentrations relativement élevées. En revanche, à des concentrations courantes, Ca perturbe le dosage de $\mathrm{Mg}$. $\mathrm{Al}$ exerce une légère action dépressive.

TABLEAU 7

Influence de quelques cations sur le dosage de $\mathrm{Mg}$.

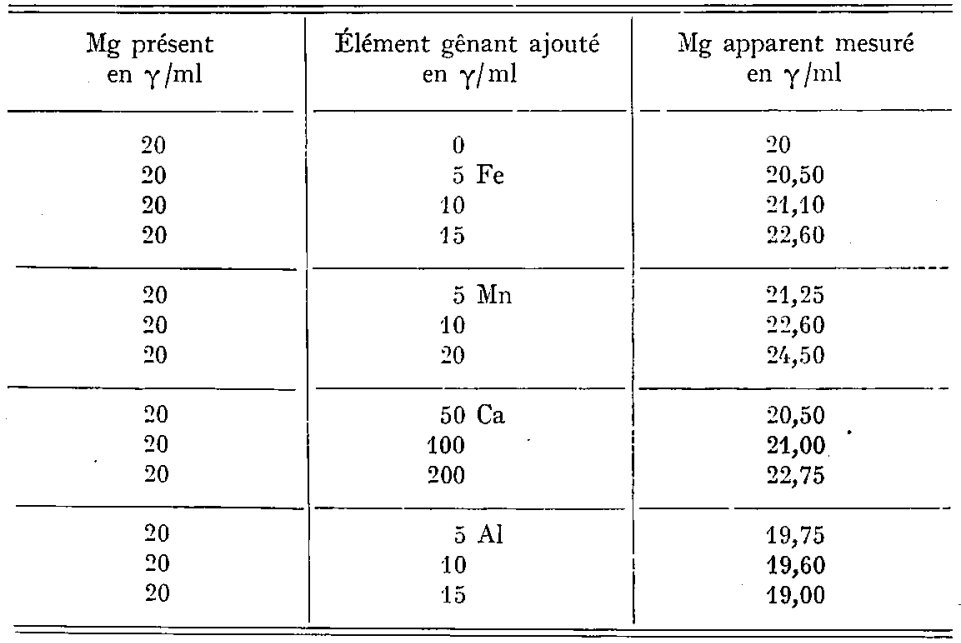


Cependant, la cause d'erreur la plus importante dans le dosage de Mg est la présence des alcalins, dont l'action excitatrice est particulièrement forte (fig. 4 et 5). Ainsi, même à des concentrations seulement égales à celles de $\mathrm{Mg}$, Na ou $\mathrm{K}$ entrâ̂nent une erreur par excès voisine de 5 p. Ioo.

Il est donc très important de connaître avec une précision suffisante la teneur

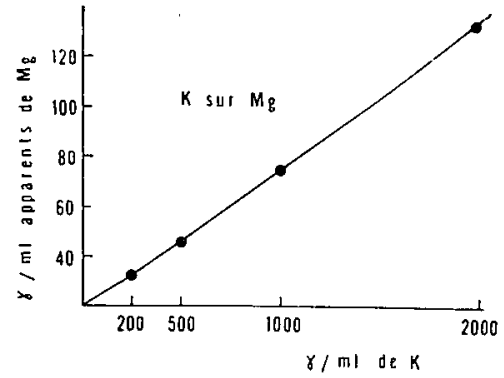

FIG. 4. -- Inlerférence due à $K$ dans le dosage de $\mathrm{Mg}$.

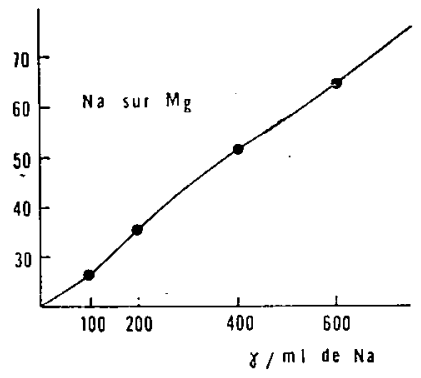

FIG. 5. - Interférence due à $\mathrm{Na}$ dans le dosage de $\mathrm{Mg}$.

en $\mathrm{K}$ et $\mathrm{Na}$ du produit à analyser et de calculer en conséquence la composition des solutions étalons. Il est également possible d'adopter, pour une certaine catégorie de produits, une composition moyenne des solutions étalons, et de calculer, sur les figures précédentes, des facteurs de correction applicables suivant les teneurs réelles en $\mathrm{K}$ et $\mathrm{Na}$ du produit. En fait, dans le cas de la plupart des aliments, set1le l'interférence due à $K$ est importante.

\section{III - APPIICATION A IA PRATIQUE DES DOSAGES}

\section{A - Préparation des solutions étalons}

Il importe de veiller tout particulièrement à la préparation des solutions étalons, et plus précisément à celle des solutions mères contenant par exemple $I \mathrm{~g}$ d'élément par litre. En effet, la pureté et surtout l'hygroscopicité de la plupart des sels est très variable : il est parfois difficile d'obtenir un sel parfaitement sec et de formule bien définie. C'est pourquoi il convient de choisir des sels purs, pouvant être chauffés à haute température sans décomposition. Ainsi, nous utilisons le carbonate de sodium anhydre, le chlorure de potassium et le carbonate de calcium (obtenu à partir de l'oxalate), après passage au four à $500^{\circ} \mathrm{C}$. En ce qui concerne le magnésium, il est difficile de préparer directement une liqueur titrée par pesée d'un sel; aussi nous utilisons le métal pur en copeaux ou en fil, que nous pesons et attaquons par de l'acide chlorhydrique, après avoir pris soin d'enlever la couche superficielle oxydée (DUVAL, I954).

Dans la préparation des solutions étalons complexes, contenant plusieurs éléments (dans le but de pallier les interférences qu'ils provoquent), il convient de veiller à ce que les conditions de milieı n'entraînent pas la précipitation de certains éléments : nous avons vu que la solubilité du perchlorate de potassium dans une solution contenant de l'acide perchlorique est bien inférieure à sa solubilité dans l'eau; 
de même, les solutions étalons de $\mathrm{Na}$ et $\mathrm{K}$ obtenues à partir des carbonates sont alcalines, ce qui peut provoquer une précipitation de $\mathrm{Ca}$ et de $\mathrm{Mg}$.

\section{$\mathrm{B}$ - Préparation des solutions à analyser}

Pour les dosages des éléments minéraux dans les aliments, (en particulier les fourrages) et dans les fécès, nous avons adopté la calcination au four à $530-55^{\circ} \mathrm{C}$ d'une prise d'essai de I g, suivie d'une insolubilisation de la silice par $5 \mathrm{ml}$ de $\mathrm{HCl}$ et d'une reprise par $\mathrm{HCl} \mathrm{I} / \mathrm{IO}(\mathrm{v} / \mathrm{v})$ our $\mathrm{HNO}_{3} \mathrm{I} / \mathrm{IO}(\mathrm{v} / \mathrm{v})$. Dans ce cas le problème de l'influence du milieu organique ne se pose pas.

En ce qui concerne le lait et l'urine, l'analyse par passage direct, après dilution convenable, serait particulièrement intéressante. Malheureusement, seule l'urine peut se prêter directement à certains dosages ( $\mathrm{K}$ et $\mathrm{Na}$ ) à la dilution de $\mathrm{I} /$ Ioo. Le lait, même à une dilution assez forte $(\mathrm{r} / 5 \mathrm{O})$ obture progressivement les canalisations et la consommation de liquide devient très irrégulière. Il est possible, en revanche, d'utiliser un lait dilué de $\mathrm{I} / 50(\mathrm{v} / \mathrm{v})$ et déprotéinisé par de 1'acide trichloracétique (selon la technique de VAN DER HAVE et MULDER I957) ; il est toutefois nécessaire de faire une correction approximative pour tenir compte du volume du précipité.

Cependant, malgré les dilutions effectuées, l'influence du milieu organique demeure mal connue et il est difficile de la compenser. Aussi est-il préférable d'effectuer une minéralisation préalable, par exemple par le mélange nitro-perchlorique pour le lait, en introduisant dans les solutions étalons une quantité convenable d'acide perchlorique. Notons également qu'il est possible d'effectuer une séparation quantitative des cations du sérum du lait par passage sur une résine échangeuse d'ions, comme Dowex 5O (WENNER, I958).

\section{$\mathrm{C}-$ Dosages}

Pour chacun des éléments étudiés, nous avons recherché les meilleures conditions de dosage à utiliser, en particulier pour ce qui concerne les pressions de gaz, les limites de sensibilité et la composition des solutions étalons. Il convient d'abord de signaler que la stabilité de la flamme, qui dépend elle-même de la pression des différents gaz utilisés, et la constance du débit de liquide, sont les conditions primordiales qui permettent d'obtenir des mesures fidèles.

\section{Io Dosage du potassium.}

- Conditions générales : le potassium est dosé dans la flamme air $\left(0,5 \mathrm{~kg} / \mathrm{cm}^{2}\right)$ propane ( $160 \mathrm{~mm}$ d'eat1). L'intervalle optimttm de concentration à utiliser semble se situer entre 5 et Ioo $\gamma / \mathrm{ml}$. Les courbes d'étalonnage obtenues sont sensiblement linéaires.

- Conditions particulières à chaque produit. Aliments, fourrages : le potassium est de loin 1'élément le plus abondant dans les fourrages, et pratiquement aucun problème d'interférence ne se pose pour son dosage. Il est nécessaire pour avoir des lectures précises de diluer 4 ou 5 fois la solution obtenue à partir des cendres (prise d'essai de I $\mathrm{g}$ de fourrage amenée à Ioo $\mathrm{ml}$ ) et d'utiliser une gamme de solutions étalons de 20 à $80 \mathrm{\gamma} / \mathrm{ml}$.

Lait : le potassium est également l'élément minéral le plus abondant dans le 
lait ; il est de ce fait inutile de composer des solutions étalons complexes pour compenser les interférences éventuelles. Le dosage s'effectue sur un lait minéralisé ou déprotéinisé dilué à $\mathrm{I} / 5^{\circ}$ et la gamme étalon est constituée de solutions à Io, 2030 et $40 \mathrm{\gamma} / \mathrm{ml}$ de $\mathrm{K}$.

Fécès : Bien que les concentrations en $\mathrm{K}$ soient inférieures à celles rencontrées dans les aliments, les taux de $K$ des fécès restent supérieurs à ceux de $\mathrm{Na}$. On n'est donc gêné par aucune interférence, mis à part les résidus acides de minéralisation $\left(\mathrm{HClO}_{4}\right.$ ou $\left.\mathrm{HCl}\right)$ et parfois le calcium. Pour une prise d'essai de $\Upsilon \mathrm{g}$ amenée à roo $\mathrm{ml}$ on pourra opérer avec des solutions étalons allant de Io à $80 \mathrm{r} / \mathrm{ml}$.

Urine: Une dilution de I/roo est en général suffisante. La lecture peut donc être directe avec des témoins purs de ro à $50 \% / \mathrm{ml}$ pour les urines des herbivores. En ce qui concerne l'urine humaine, ou celle des omnivores, les concentrations de Na pouvant être doubles de celles de $K$, il est nécessaire de doser $\mathrm{Na}$ en premier et de tenir compte de cette teneur pour les solutions étalons.

\section{$2^{\circ}$ Dosage du sodium :}

La flamme utilisée et les pressions de gaz sont les mêmes que pour K. Les concentrations optima de dosage sont comprises entre 2 et $50 \mathrm{\gamma} / \mathrm{ml}$ et les courbes étalons s'infléchissent légèrement pour les concentrations élevées.

Fourrages: Compte tenu de l'influence de $\mathrm{K}$ dans le dosage de $\mathrm{Na}$ et de la grande variabilité du rapport $\mathrm{K} / \mathrm{Na}$ dans les fourrages, il est nécessaire de posséder plusieurs séries de solutions étalons suivant la teneur présumée en $\mathrm{Na}$ (celle-ci peut varier de $\mathrm{o,I}$ à $8 \mathrm{p}$. IOOo). Il est également nécessaire de choisir une dilution convenable, ou une amplification permettant d'effectuer des lectures précises. Il est possible, au besoin, d'utiliser le photomultiplicateur plus sensible destiné au dosage de $\mathrm{Mg}$.

Lait : le rapport $\mathrm{K} / \mathrm{Na}$ moyen du lait de vache est de 3 ; il suffit donc de composer des solutions étalons ayant ce même rapport moyen, du moins pour des laits de mélange (le rapport $\mathrm{K} / \mathrm{Na}$ des laits individuels est susceptible de varier considérablement suivant le stade de lactation et la présence d'une infection microbienne de la mamielle).

Fécès : les teneurs en $\mathrm{K}$ sont supérieures à celles en $\mathrm{Na}$. On doit par conséquent ajouter du $\mathrm{K}$ dans les solutions étalons de dosage de $\mathrm{Na}$, mais on peut se contenter d'une seule gamme de 5 à $50 \gamma / \mathrm{ml}$ de $\mathrm{Na}$ contenant la teneur moyenne en $\mathrm{K}$ des solutions de fécès.

Urines: Dans les urines des herbivores, la teneur en $\mathrm{Na}$ étant faible, il est nécessajte d'ajouter dans les témoins de dosage une quantité de $\mathrm{K}$ équivalente à celle des échantillons. Bien que l'erreur absolue soit faible, l'erreur relative peut être très importante sur de si basses teneurs. Une dilution de $\mathrm{r} / \mathrm{roo}(\mathrm{v} / \mathrm{v})$ permet de travailler entre 5 et $20 \mathrm{\gamma} / \mathrm{ml}$ de $\mathrm{Na}$. Pour les urines des omnivores le potassium ne gêne en général pas ; il est souvent nécessaire, en revanche, d'effectuer des dilutions plus importantes, $\mathrm{I} / 200(\mathrm{v} / \mathrm{v})$ par exemple.

\section{$3^{\circ}$ Dosage du calcium:}

- Conditions générales - Le calcium est excité à une température plus élevée (la flamme étant donc elle-même un moyen de sélection des éléments), supérieure à $2000^{\circ} \mathrm{C}$ et son dosage s'effectue dans la flamme air $\left(0,5 \mathrm{~kg} / \mathrm{cm}^{2}\right.$ - acétylène $(500 \mathrm{~mm}$ d'eau ) et éventuellement oxygène ( 2 litres/mn). En fait nous avons constaté que les 
meilleurs résultats, en particulier un minimum d'interférence due au phosphore, étaient obtenus en utilisant la flamme acétylène-air. D'autre part, l'emploi d'oxygène diminue la sensibilité des mesures et les variations de son débit constituent une cause supplémentaire d'erreur. Les meilleures conditions de dosage sont réalisées pour des concentrations de 20 à Ioo $\gamma / \mathrm{ml}$; dans ces conditions les courbes d'étalonnage sont en général linéaires.

\section{- Conditions particulières à chaque produit}

Fourrages : Pour la confection des solutions étalons il est nécessaire de dissocier les graminées et les légumineuses (en moyenne 4 fois plus riches en $\mathrm{Ca}$ ) et, en particulier pour les graminées, de tenir compte de l'interférence due à $\mathrm{K}$, le rapport $\mathrm{K} / \mathrm{Ca}$, pouvant atteindre des valeurs élevées. Notons également que pour d'autres aliments, en particulier les composés minéraux, il y a lieu de tenir compte dans le dosage du calcium des interférences dues à l'aluminium et au fer, et rappelons que l'erreur due à la présence de l'aluminium peut être pratiquement négligée quand on ajoute dans toutes les solutions un excès de strontium (par exemple $2000 \gamma$ par ml).

Pour les fourrages composés en majeure partie de graminées, le dosage peut se faire sur la solution obtenue en complétant à roo $\mathrm{ml}$ le résidu de calcination de I $\mathrm{g}$ de produit, en utilisant une gamme de Io à $50 \% / \mathrm{ml}$ de Ca. En revanche, pour les légumineuses, il est nécessaire de diluer 4 ou 5 fois la solution précédente.

Lait : La composition minérale du lait, en particulier sa teneur en $\mathrm{Ca}$, est relativement constante ; en conséquence, le dosage du Ca dans un lait dilué 50 fois, minéralisé ou déprotéinisé, ne présente pas de difficultés, la gamme étalon allant de Io à $50 \gamma / \mathrm{ml}$ de $\mathrm{Ca}$. Aucune interférence n'est à craindre.

Fécès : les fécès sont plus riches en calcium qu'en potassium, surtout chez les herbivores où l'excrétion se fait presque uniquement par la voie intestinale. Le potassium n'est donc pas un élément gênant. En revanche, chez des animaux ayant reçu un condiment minéral contenant du sulfate de magnésium, on peut avoir une légère interférence de cet élément sur le dosage du calcium. En pratique, I g de produit sec amené à Ioo $\mathrm{ml}$ permet les dosages entre 20 et Ioo $\gamma / \mathrm{ml}$ après une dilution de $\mathrm{I} / 5$.

Urines : le dosage du calcium dans les urines des herbivores est délicat. Les quantités excrétées sont en effet particulièrement faibles, de 0,02 à $0,05 \mathrm{~g}$ par litre, tandis que ces urines contiennent de 4 à $5 \mathrm{~g}$ de $\mathrm{K}$ par litre, environ $\mathrm{I}, 2 \mathrm{~g}$ de $\mathrm{Na}$ et de 0 , ro à $0,25 \mathrm{~g}$ de $\mathrm{Mg}$. Eiffectué avec des solutions étalons pures de $\mathrm{Ca}$, l'erreur sur le dosage serait de $-4 \mathrm{p}$. Ioo pour un rapport $\mathrm{K} / \mathrm{Ca}$ de Ioo et de $-6,5 \mathrm{p}$. Ioo pour $\mathrm{Mg} / \mathrm{Ca}$ de ro (voir dosage de $\mathrm{Ca}$ ). En revanche, $\mathrm{Na}$ n'interfère pas sur le dosage de $\mathrm{Ca}$. Il est donc nécessaire d'ajouter dans les solutions étalons des quantités de $\mathrm{K}$ et de $\mathrm{Mg}$ voisines de celles rencontrées dans les échantillons. Étant donné la grande variabilité des teneurs en $\mathrm{Mg}$ de l'urine, on devra établir au moins deux gammes de solutions étalons de $\mathrm{Ca}$, l'une pour les faibles teneurs en $\mathrm{Mg}$ (de 0, Io à $0, \mathrm{I} 7 \mathrm{~g}$ par litre par exemple) et l'autre pour les teneurs plus fortes (0,17 à $0,25 \mathrm{~g}$ par litre).

Le seul dosage de Ca urinaire entraine donc automatiquement le dosage des autres éléments, $\mathrm{Na}, \mathrm{K}$ et $\mathrm{Mg}$. En effet, l'erreur due à $\mathrm{Mg}$ étant la plus importante, sa teneur doit être bien connue, et l'on sait que son dosage nécessite la connaissance des teneurs en $\mathrm{K}$ et $\mathrm{Na}$. De plus, si les teneurs en $\mathrm{K}$ ćtaient trop variables d'un échantillon à 1'autre, on pourrait être amené à utiliser 4 gammes de solutions étalons, combinant des teneurs fortes et faibles en $\mathrm{K}$ et $\mathrm{Mg}$. 
4" Dosage du magnésium:

- Conditions générales - Le magnésium est dosé dans une flamme chaude, comme celle obtenue par l'hydrogène ( $800 \mathrm{~mm}$ d'eau), l'oxygène (2 litres $/ \mathrm{mn})$ et l'air $\left(0,5 \mathrm{~kg} / \mathrm{cm}^{2}\right)$. Il est nécessaire d'utiliser un brûleur spécialement conçı pour l'emploi de 1'hydrogène. D'autre part, l'émission de $\mathrm{Mg}$ étant mesurée dans l'ultra-violet, il faut utiliser un autre photomultiplicateur particulièrement sensible; en général il est difficile d'obtenir tune stabilité parfaite du spot lumineux et il importe donc de choisir l'amplification la moins forte possible, compatible avec les concentrations en $\mathrm{Mg}$ dans le produit à analyser, permettant d'obtenir une fidélité satisfaisante.

Signalons également que la forte pression utilisée pour l'hydrogène provoque l'apparition de bulles d'air dans la canalisation de sortie, et rend le débit irrégulier. Il suffit alors de diminuer le débit de sortie du liquide. Enfin, il importe de s'assurer de la parfaite stabilité des pressions de 1'hydrogène et de l'oxygène; pour cela l'emploi de microdétendeurs à la sortie des bouteilles est indispensable. Les meilleures conditions de mesure sont obtenues pour des concentrations de 50 à $200 \mathrm{\gamma} / \mathrm{ml} \mathrm{de} \mathrm{Mg}$; les courbes obtenues sont alors linéaires. Cependant il est possible de mesurer dans des conditions satisfaisantes des concentrations aussi faibles que Io $\gamma / \mathrm{ml} \mathrm{de} \mathrm{Mg}$.

Conditions particulières à chaque dosage - Nous avons vu que le potassium et le sodium sont les éléments les plus gênants dans le dosage du magnésium. Aussi est-il indispensable, quel que soit le produit à analyser, de connaître avec tune bonne précision ses teneurs en $\mathrm{K}$ et $\mathrm{Na}$, a fin de pouvoir utiliser des solutions étalons de composition voisine. Il suffit en général d'employer des solutions témoins de composition moyenne, et d'effectuer une correction calculée d'après la teneur exacte dư produit en $\mathrm{K}$ et $\mathrm{Na}$.

Il est également possible d'utiliser la méthode des surcharges, en tenant compte dans les calculs de la valeur effectivement trouvée pour la surcharge ajoutée à la'solution.

Aliments : Dans les fourrages, la seule interférence importante est celle due à $\mathrm{K}$, et il est indispensable d'en tenir rigoureusement compte. Cependant certaines graminées fourragères (en particulier le dactyle) semblent plus pauvres en $\mathrm{Mg}$ qu'en $\mathrm{Na}$ etl 'influence de $\mathrm{Na}$ devient alors importante. D'autre part, dans le cas des légumineuses, l'action de Ca n'est pas négligeable, et il faut également en introduire dans les solutions étalons. La dilution obtenue en amenant à $50 \mathrm{ml}$ une prise d'essai de I g de fourrage permet d'effectuer le dosage dans de bonnes conditions.

Dans l'analyse de certains composés minéraux, il y a lieu de tenir compte d'autres interférences dues au fer, à l'aluminium,'au manganèse, et bien entendu au sodium.

Lait : le lait de vache est pauvre en $\mathrm{Mg}$, et son dosage est ainsi gêné par plusieurs interférences, en particulier celles dues au potassium, au sodium et au calcium. Connaissant la composition moyenne du lait de vache, il suffit cependant de préparer des solutions étalons dont les rapports moyens soient de Io pour $\mathrm{Ca} / \mathrm{Mg}$, de $\mathrm{x} 2$ pour $\mathrm{K} / \mathrm{Mg}$ et de 5 pour $\mathrm{Na} / \mathrm{Mg}$.

Afin d'effectuer les mesures dans des conditions satisfaisantes, le dosage de $\mathrm{Mg}$ -doit être réalisé đans un lait dilué de $\mathrm{I} / \mathrm{IO}(\mathrm{v} / \mathrm{v})$ seulement et, à cette dilution, la minéralisation s'impose. Iin effet, le lait simplement déprotéinisé et dilué de $\mathrm{I} / \mathrm{Io}(\mathrm{v} / \mathrm{v})$ ne permet pas une bonne pulvérisation et les résultats obtenus sont peu valables. Une :gamme de solutions étalons de Io à $20 \mathrm{\gamma} / \mathrm{ml} \mathrm{de} \mathrm{Mg}$ convient dans tous les cas. 
Fécès : Bien que les qua:atités de $\mathrm{K}$ ne soient que de 4 à $6 \mathrm{mg}$ par gramme de produit sec, c'est-à-dire équivalentes à celles de $\mathrm{Mg}$, et que le rapport $\mathrm{Mg} / \mathrm{Na}$ soit de 2 à 4, l'interférence des alcalins, même à ces faibles concentrations, n'est pas négligeable. On devra donc en tenir compte pour la composition des témoins. Eitant donné la faible sensibilité du dosage de $\mathrm{Mg}$, il faudra le plus souvent travailler directement sur le liquide de minéralisation ( $\mathrm{g}$ de produit $\mathrm{sec}$ amené à roo $\mathrm{ml}$ ), sans dilution avant le dosage. On devra donc ajouter dans les solutions étalons, en plus de $\mathrm{K}$ et de $\mathrm{Na}$, des quantités d'acides équivalentes à celles restant dans le liquide de minéralisation. Il est nécessaire également de vérifier qu'au cours des récoltes les échantillons de fécès n'ont pas été souillés par du fer ou de l'aluminium, ce qui fausserait le dosage du magnésium. On peut, en général, travailler avec des solutions étalons de 30 à IOO $\gamma / \mathrm{ml}$.

Urines : Les fortes concentrations en alcalins rendent particulièrement difficile le dosage du magnésium urinaire (figures 4 et 5 ). Comme l'indique la composition de l'urine des herbivores (rapportée dans l'étude du dosage du calcium urinaire) le rapport de concentration des alcalins au magnésium est de 25 à 50 pour le potassium et de 6 à I2 pour le sodium. Dans ces conditions, un dosage effectué avec des témoins purs entrainerait des erreurs par excès de I25 p. Ioo environ pour le potassium et de $85 \mathrm{p}$. Ioo pour le sodium. On devra donc obligatoirement doser au préa- lable $\mathrm{Na}$ et $\mathrm{K}$. La technique la plus sûre consiste à établir une courbe étalon pour chaque échantillon. L'erreur est ainsi ramenée à une valeur systématique de $+4 \mathrm{p}$. Ioo, donc de correction facile (cf. tableau 9). Dans le cas des dosages en série, où il est impossible de multiplier les gammes étalons, on pourra opérer de la façon suivante :

- doser $\mathrm{Na}$ et $\mathrm{K}$.

- établir 4 gammes de solutions étalons combinant les teneurs moyennes, faibles ou fortes, en alcalins des échantillons (comme pour le dosage de $\mathrm{Ca}$ urinaire).

- tracer les courbes d'interférences de $\mathrm{Na}$ et de $\mathrm{K}$ sur le dosage de $\mathrm{Mg}$ à partir de ces valeurs moyennes utilisées dans les témoins.

- avec une dilution de l'urine au $\mathrm{I} / 4(\mathrm{v} / \mathrm{v})$ on pourra ainsi doser $\mathrm{Mg}$ avec des solutions étalons s'échelonnant de 20 à Ioo $\gamma / \mathrm{ml}$.

On aura donc deux corrections successives :

- Au moment du dosage, par l'emploi des témoins complexes, contenant des quantités de $\mathrm{K}$ et $\mathrm{Na}$ assez voisines de celles des échantillons.

- Mais dans la plupart des cas cette correction est insuffisante. Ainsi, par exemple, si les teneurs en $\mathrm{Na}$ et $\mathrm{K}$ des échantillons à analyser s'écartent de ro p. Ioo des. valeurs moyennes choisies pour les solutions étalons, l'erreur commise sur la teneur en $\mathrm{Mg}$ est déjà de 1'ordre de $5 \mathrm{p}$. Ioo. On devra donc, à l'aide des courbes d'interférences, appliquer une correction à la valeur obtenue par dosage.

\section{D. - Contrôle de la validité des résultats obtenus}

Deux moyens de contrôle peuvent être utilisés, à savoir : les essais de surcharges. et la comparaison aux méthodes chimiques réputées spécifiques.

Des essais de surcharges ont été effectués pour la plupart des dosages. Les résultats varient bien entendu suivant l'importance des interférences et la similitude plus ou moins grande qui existe entre les compositions des solutions étalons et de la solution à analyser. 
Les tableaux 8 et 9 rassemblent les résultats de deux essais de surcharges: 1'un concerne le dosage de $\mathrm{Ca}$ dans des fécès après calcination, avec comparaison à des solutions étalons pures, l'autre le dosage de $\mathrm{Mg}$ dans l'urine, avec comparaison à des solutions étalons complexes, contenant les éléments interférents à des concentrations proches de celles de l'urine.

\section{TABLEAU 8}

Essais de surcharges de calcium sur fécès.

(Mesures effectuées anec solutions élalon pures).

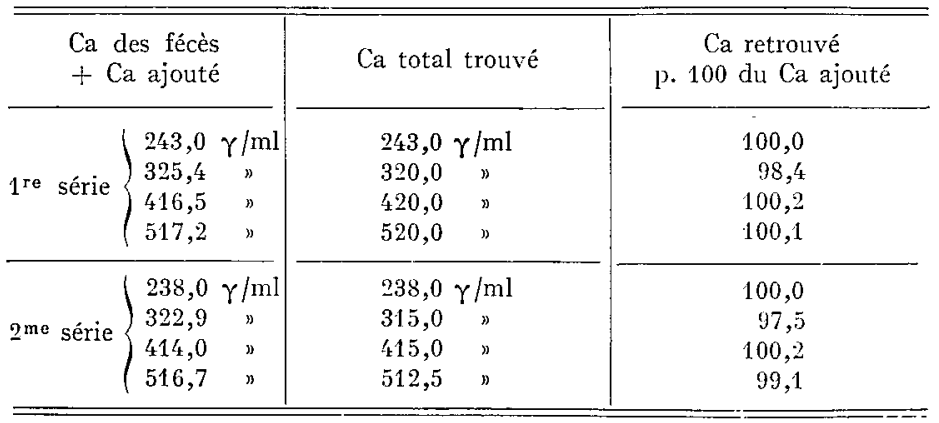

TABLEAU 9

Essais de surcharges de $M g$ sur urine.

(Mesures effectuées avec solutions étalon compensées).

\begin{tabular}{|c|c|c|}
\hline $\begin{array}{l}\mathrm{Mg} \text { de l'urine } \\
+\mathrm{Mg} \text { ajouté }\end{array}$ & Mg total trouvé & $\begin{array}{l}\text { Mg retrouvé } \\
\text { en p. } 100 \text { du } \mathrm{Mg} \text { ajouté }\end{array}$ \\
\hline $70 \gamma / \mathrm{ml}$ & $71,0 \mathrm{\gamma} / \mathrm{ml}$ & $101,4_{4}$ \\
\hline $80 "$ & $82,0 \quad$ & 103,7 \\
\hline 90 & $93,2 》$ & 103,6 \\
\hline 100 & 101,2 & 101,2 \\
\hline 110 & 113,2 & 102,9 \\
\hline 120 & 135,2 " & $10^{\prime}, 3$ \\
\hline
\end{tabular}

Ainsi, dans un dosage ot̀ l'on risque peu d'être gêné par des interférences, par exemple le dosage du calcium dans les fécès, l'emploi de solutions étalons pures permet de trouver pratiquement la totalité des surcharges ajoutées. Cependant, dans un dosage particulièrement délicat comme celui du magnésium dans l'urine, les surcharges sont retrouvées avec une erreur systématique relativement faible, à condition d'utiliser des solutions étalons de compositions aussi proches que possible de celle de l'urine.

Pour résumer les comparaisons effectuées à l'occasion de "chaînes " d'analyses, en collaboration avec de nombreux laboratoires français et étrangers utilisant des méthodes chimiques, nous rapportons dans le tableau Io les résultats obtenuts par l'emploi du photomètre à flamme "Eppendorf » et la moyenne des résultats fournis par plusieurs méthodes chimiques et colorimétriques courantes. 
TABLEAU IO

Comparaison des résultats obtenus par photomélrie de flamme et par des méthodes chimiques.

\begin{tabular}{|c|c|c|c|c|c|c|c|c|}
\hline & \multicolumn{4}{|c|}{$\begin{array}{l}\text { Photométrie de flamme } \\
\text { résultats en } \mathrm{g} \text { pour } 100 \mathrm{~g}\end{array}$} & \multicolumn{4}{|c|}{$\begin{array}{l}\text { Méthodes chimiques } \\
\text { résultats en } \mathrm{g} \text { pour } 100 \mathrm{~g}\end{array}$} \\
\hline & $\mathrm{Ca}$ & $\mathrm{K}$ & $\mathrm{Na}$ & $\mathrm{Mg}$ & $\mathrm{Ca}$ & $\mathrm{K}$ & $\mathrm{Na}$ & $\mathrm{Mg}$ \\
\hline Luzerne 1 & 1,69 & 3,01 & 0,05 & $0,3 \stackrel{2}{2}$ & 1,68 & 3,05 & 0,06 & 0,29 \\
\hline Luzcrne 2 & 1,26 & 1,98 & 0,25 & 0,23 & 1,27 & 2,12 & 0,26 & 0,21 \\
\hline Fétuque .. & 0,40 & 2,34 & 0,096 & 0,17 & 0,45 & 2,25 & 0,030 & 0,13 \\
\hline Graminée... & 0,70 & 3,15 & 0,30 & - & $0,7^{\prime} \mathrm{k}$ & 3,26 & 0,35 & -- \\
\hline Luzerne 3 . & 1,39 & 3,38 & 0,07 & 0,31 & 1,42 & $3, \overline{5} 4$ & 0,08 & $0,2 \underline{4}$ \\
\hline Luzerne 4. & 1,33 & 3,26 & 0,05 & 0,19 & 1,33 & $3,1^{\prime}$ & 0,06 & 0,17 \\
\hline Dactyle $\ldots \ldots \ldots$ & 1,11 & 2,10 & 0,12 & 0,24 & $1,11_{t}$ & 2,08 & 0,12 & 0,25 \\
\hline
\end{tabular}

Les résultats obtenus pour $\mathrm{Mg}$ sont les seuls à ne pas être rigoureusement comparables, ceci provenant également du fait que les méthođes chimiques utilisées ne sont pas elles-mêmes parfaitement spécifiques.

En conclusion, seul le dosage de $\mathrm{Mg}$ dans certains produits très riches en alcalins (liquides biologiques) présente des difficultés parfois insurmontables. Ces problèmes, soulevés dans le cas de 1'urine, se posent encore plus dans l'analyse du sérum sanguin. Nous aborderons prochainement cette étude en ayant recours à des séparations préalables, soit par précipitation, soit par passage sur colonnes échangeuses d'ions.

Il importe enfin d'insister sur le fait que l'interférence globale due au milieu n'est pas égale à la somme algébrique des interférences dues à chacun de ses éléments séparés. Cependant, le milieu complexe analysé, quand il ne nuit pas à la pulvérisation dans la flamme, estéen général favorable à l'exactitude des mesures, en exerçant un certain effet tampon vis à vis des interférences particulières. Bien entendu, cette étude ne fait qu'aborder les multiples problèmes que pose 1'emploi de la spectrophotométrie de flamme dans un domaine particulier, mais une mise au point approfondie pour chaque appareil et pour chaque substance se justifie pleinement par la rapidité et la sensibilité de ces méthodes.

Rę̧u en janvier 1960.

\section{SUMMARY}

ESTIMATION BY FLAME SPECTROPHOTOMETRY OF SODIUM, POTASSIUM, CALCIUM AND MAGNESIUM IN FEEDSTUFFS, MILK AND EXCRETA.

The "Eppendorf " flame photometer offers new possibilities for the determination of alkalis and alkaline earths in biological materials. In addition to sodium and potassium, the estimation of calcium can be carried out under very good conditions, with an acetylene flame, interference filter and photomultiplier tube. The determination of magnesium, which is much more delicate, can, however, be performed using a very sensitive photomultiplier and a hydrogen flame whose own emission is weak at low wave lengths.

We have studied the main interfering factors likely to affect the determinations, in particular the influence of organic media, anions and cations. Although particularly selective, this photometer does not eliminate all these interferences. Thus potassium affects all the estimations, and in 
particular the determination of magnesium : for example, the errors introduced are +6 p. 100 in estimating $\mathrm{Na}$ for a $\mathrm{K} / \mathrm{Na}$ ratio of 5 , of $-4 \mathrm{p}$. 100 in the estimation of $\mathrm{Ca}$ for a $\mathrm{K} / \mathrm{Ca}$ ratio of 4 , and $+20 \mathrm{p} .100$ in the estimation of $\mathrm{Mg}$ for a $\mathrm{K} / \mathrm{Mg}$ ratio of 5 . Likewise, sodium considerably enhances the $\mathrm{Mg}$ emission, and the error can reach $+25 \mathrm{p}$. Ioo for an $\mathrm{Na} / \mathrm{Mg}$ ratio of 3 .

On the other hand, the effect of calcium on the determination of $\mathrm{K}$ and $\mathrm{Na}$ is low, and surprisingly enough the influence of phosphorus on the estimation of $\mathrm{Ca}$ is negligible.

These interferences being independent of the concentration of the elements to be determined, we have adopted the technique of compensated standard solutions, whose concentrations of disturbing elements correspond to the average concentrations of the solutions to be analysed. Thus we have defined the optimal conditions required for the analysis of fodder, milk, faeces and urine, by specifying the main sources of error encountered and by justifiyng the validity of the results obtained.

The determinations are carried out with fodder and faeces after ashing the material, and with milk and urine after nitro-perchloric digestion. Only the determination of magnesium in urine presents serious difficulties owing to its high alkali content and, as in the case of blood, it is preferable, in order to obtain good accuracy, to separate first the magnesium from the alkaline metals.

\section{RÉFÉRENCES BIBLIOGRAPHIQUES}

BAKER G. L., Johnson L. H., 1954. Effects of anions on calcium flame emission in flame photometry. Analyt. Chem., 26, 465-468.

Burriel-Marti F., Ramirez-Nunoz J., I957. Flame photometry. Elsevier publishing comp. AnsterdamLondon, $54 \mathrm{I} \mathrm{pp}$.

Duval C., I954. Traité de microanalyse minérale. Presses scientifiques Internat. Paris, $1,552 \mathrm{pp}$.

Have A. J., van der, Mulder H., I957. Flame photometric estimation of the sodium, potassium and calcium content of milk and cheese. Netherl. Milk Dairy J., 11, r28-I43.

Mavrodineau R., Borteux H., I954. L'analyse spectrale quantitative par la flamme. Masson et Cie Edit. Paris, $207-222$.

Mitcielel R. L., Robertson I. M., I 936. The effect of aluminium on the flame spectra of the alkaline earths : a method for the determination of aluminium. J. Soc. Chem. Ind. Lond., 55, 269 T.

PinTa M., Bove C., 1956. Sur la réduction des erreurs dans l'analyse végétale par spectrophotométrie de flamme. C. R. Acad. Sci., 243, r79-81.

WENNER V. R., I 958 . Rapid determination of milk salts and ions. I .Determination of sodium, potassium, magnesium and calcium by flame spectrophotometry. J. Dairy Sci., 41, 761-68. 